\title{
A TECHNIQUE FOR THE MEASUREMENT OF HOT SPOTS AND ISOTHERM PROFILES AT THE SURFACES OF THE ELEMENTS OF HYBRID MICROCIRCUITS
}

\author{
F. N. SINNADURAI \\ British Post Office Research Centre, Martlesham Heath, Ipswich, IP5 7RE
}

(Received June 19, 1979)

\begin{abstract}
A simple technique for measuring surface temperatures with high sensitivity and spatial resolution, which is particularly useful for examining temperature distributions of microcircuits, is described. The invention exploits the well-defined "Isotropic-point" transitions of nematic liquid crystals from optical birefringence to anisotropy, using a polarising microscope to detect temperatures in microscopic areas. Thus hot spots appear black against a bright background. The temperature sensitivity is better than $0.5^{\circ} \mathrm{C}$ and the spatial resolution better than 5 microns. The technique has distinct advantages over alternative methods of measuring microcircuit temperatures. Examples of applications in support of design evaluation and fault diagnosis of monolithic circuits illustrate the benefits of visual information obtained. The ready application of the technique to the large planar areas of thick film resistors has enabled the location of hot spots - confirming their occurrence at the end of laser cuts - and the measurement of thermal resistances of resistors either singly or in arrays - showing that substantial heat conduction through the substrate can outweigh local non-uniformities in dissipation. Applications extend beyond the supportive role to reliability evaluation illustrated in this paper.
\end{abstract}

\section{INTRODUCTION}

The temperatures of microelectronic components can significantly influence their performance and reliability; but their internal temperatures are rarely uniform, instead being distributed according to the power dissipation and heat conduction paths - which can follow the microscopic dimensions of some active microcircuits. Therefore techniques have been sought to measure temperatures and thermal profiles on a microscopic scale.

One of the early innovations was the infrared microradiometer, which measured the radiation emitted from microscopic areas. ${ }^{1,2}$ In principle the technique had a spatial resolution down to $8 \mu \mathrm{m}$ and a temperature sensitivity of $0.5^{\circ} \mathrm{C}$. In practice the technique rarely performed to its limits and suffered the drawback of needing considerable calibration and computation to translate the measured radiation into temperature. Liquid crystals were examined following reports of the use of cholesterogens to measure surface temperatures ${ }^{3}$ but the technique was found to have a poor spatial resolution $(>20 \mu \mathrm{m})$ and the required non-reflecting coating impaired the temperature profiles. Instead, a property of nematic liquid crystals (nematogens) has been successfully exploited ${ }^{4,5}$ into a technique for the measurement of surface temperatures with a spatial resolution of better than $5 \mu \mathrm{m}$ and sensitivity of better than $0.5^{\circ} \mathrm{C}$. The technique is briefly described in this paper which is devoted more to illustrating its application to active and passive components that could comprise the elements of hybrid microcircuits.

\section{THE TECHNIQUE}

In its liquid state, a nematogen is birefringent at temperatures up to a well defined critical temperature $\left(T_{i}\right)$ called its "Isotropic Point," above which it is isotropic. Thus, plane-polarised light is doubly refracted when transmitted through a nematogen below $T_{i}$, but is unaltered by a nematogen above $T_{i}$. In the application of the technique to microcircuits, a metallurgical microscope is employed in the experimental arrangement shown in Figure 1. A planar component coated with a thin layer $(\approx 5 \mu \mathrm{m})$ of liquid crystal is illuminated with vertically-incident plane-polarised light and viewed through a "crossed" analyser. Regions of the component at temperatures below $T_{i}$ remain visible because one of the components of the doubly-refracted light is trans- 


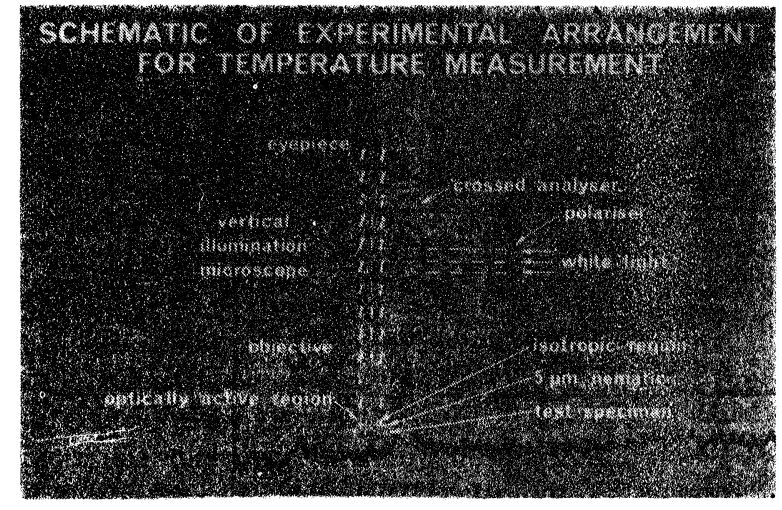

FIGURE 1 Schematic of experimental arrangement for temperature measurement.

mitted through the analyser; while regions above $T_{i}$ appear dark because light is absorbed by the analyser. The boundaries between dark and light regions are then isotherms at $T_{i}$, which has been found to be reproducible to within $0.5^{\circ} \mathrm{C}$, whilst the spatial resolution has been determined to be better than $5 \mu \mathrm{m} .{ }^{4}$ The high resolution is simply illustrated in the example shown in Figure 2 of a hot spot of about $5 \mu \mathrm{m}$ diameter, produced by dissipation in a thin-film nichrome resistor.

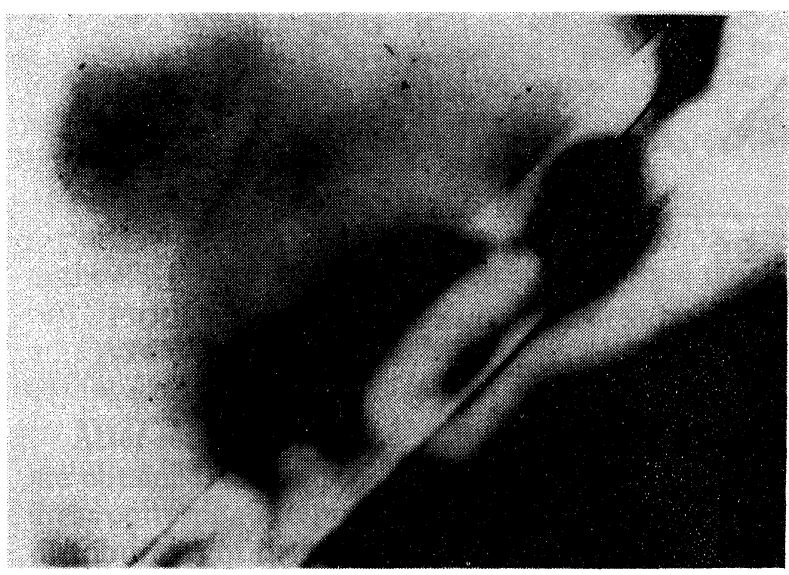

FIGURE 2 Hot spot on thin-film nichrome resistor (10 $\mu \mathrm{m}$ wide).

In order to measure temperatures below $T_{i}$, for a particular dissipation, the component ambient temperature $\left(T_{a}\right)$ is increased until light is extinguished in the area of interest. Then $T_{i}-T_{a}$ is the temperature rise for that dissipation. The distribution of temperatures between $T_{i}$ and $T_{a}$ is obtained by raising $T_{a}$ by small increments to

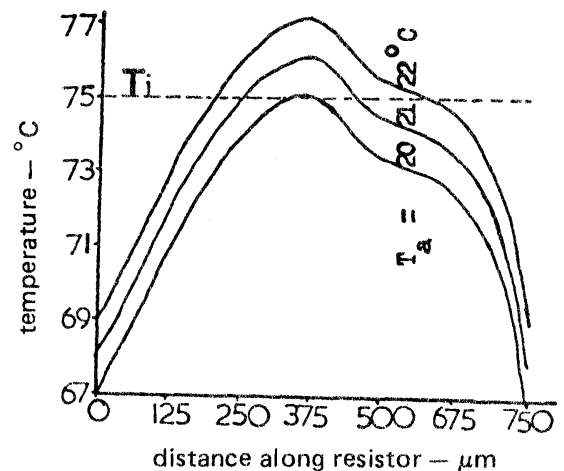

FIGURE 3 Temperature distribution along resistor of Figure. 2 .

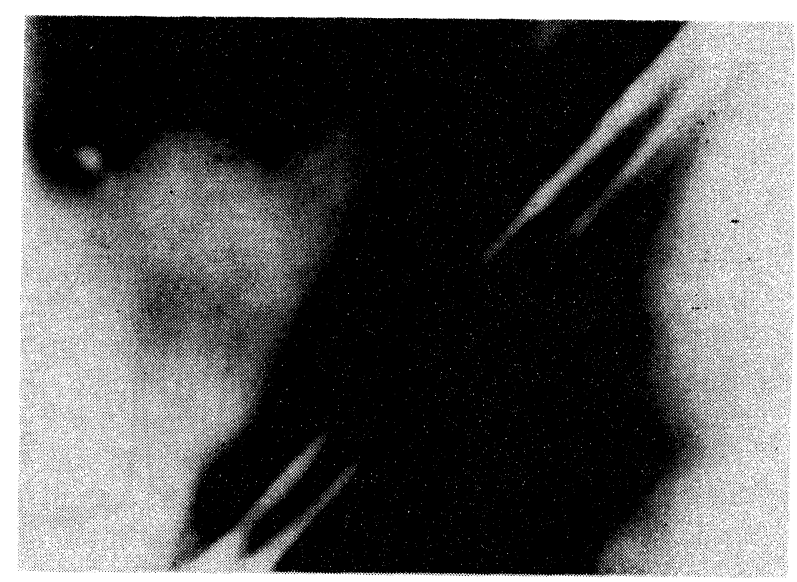

FIGURE 4 Isothermal boundaries extended by raising ambient by $1^{\circ} \mathrm{C}$.

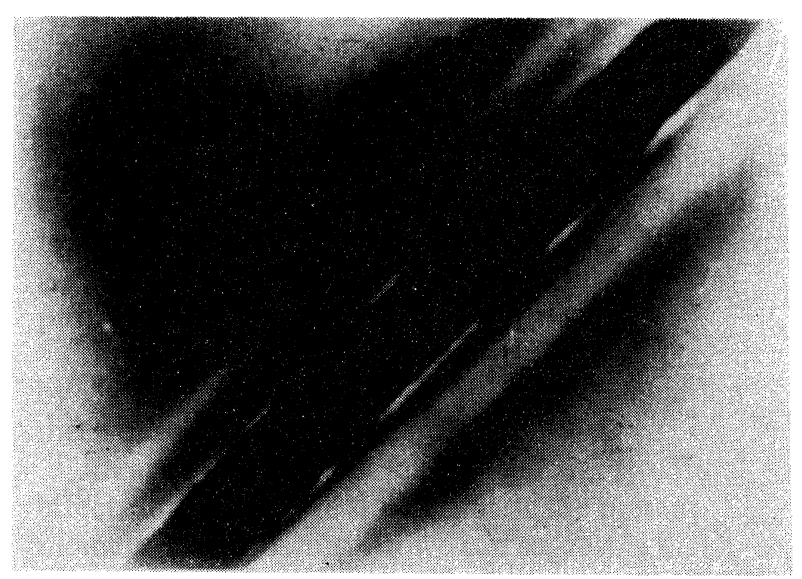

FIGURE 5 Isotherm boundaries extended by raising ambient by $2^{\circ} \mathrm{C}$. 
produce a succession of isotherm boundaries corresponding to each $T_{i}-T_{a}$. The temperature distribution in the resistor from the earlier example was obtained in this way (Figure 3). Figure 3 also illustrates the manner in which the areas bounded by the isotherms widen as $T_{a}$ is increased by $1^{\circ} \mathrm{C}$ and $2^{\circ} \mathrm{C}$, and the corresponding effects are illustrated in Figures 4 and 5. Temperatures above the isotropic point of a particular nematogen may be measured either by cooling the ambient or by using nematogens with a higher $T_{i}$. Nematogens have been identified with isotropic points in the range $25^{\circ} \mathrm{C}$ to $300^{\circ} \mathrm{C}$.

\section{APPLICATIONS}

\subsection{Monolithic Semiconductor Components.}

The technique is especially suited to monolithic microcircuits and transistors, where the high resolution is exploited. The following examples have been chosen to illustrate the obvious benefits of the immediate visual information obtained.

The first example shows the operation of an interdigitated transistor, clearly revealing, in Figure 6,

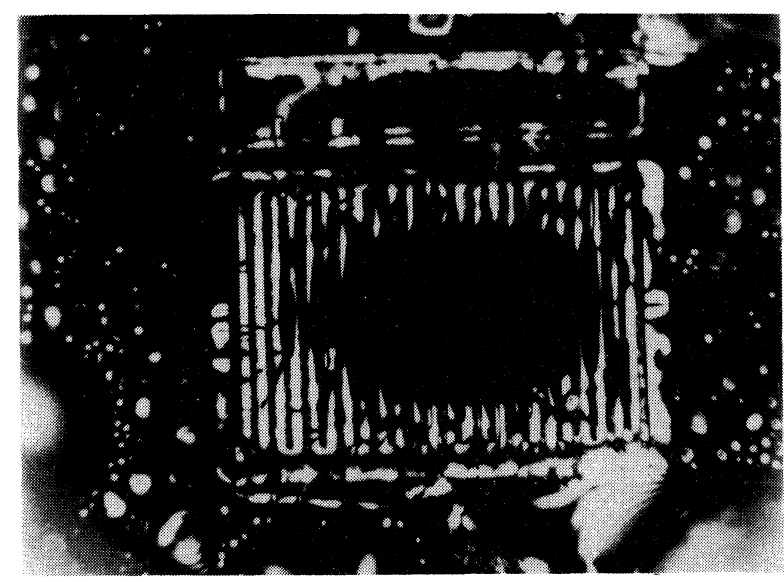

FIGURE 6 Hotter regions of one segment of an interdigitated transistor.

the satisfactorily symmetrical distribution of temperature about the centre of one segment. The outer elements just reaching $T_{i}$ are clearly distinguishable. Figure 7 shows that one entire segment of the transistor was malfunctioning requiring excessive dissipation in the other three segments to get the fourth up to operating temperatures.

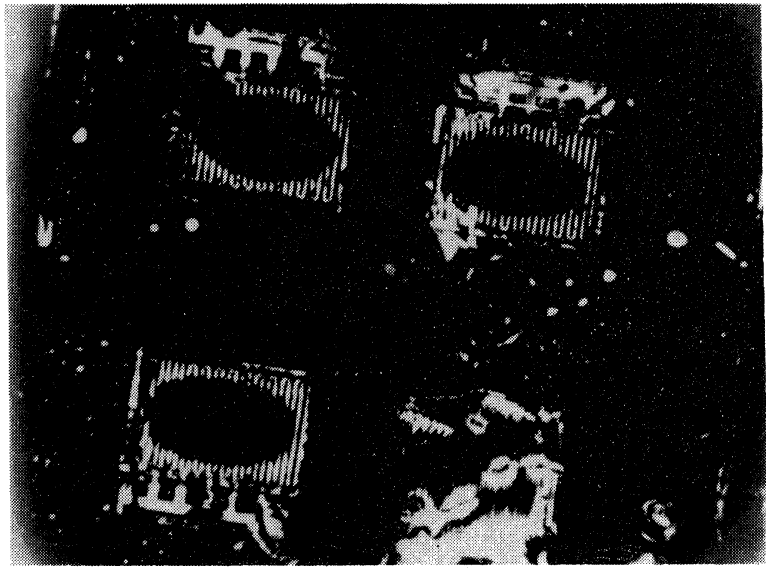

FIGURE 7 Malfunction revealed in one segment of the transistor.

A second example is of the diagnosis of faulty relay driver transistors (Figure 8 ) of a type that frequently failed under transient surge conditions. Figure 9 shows the hotter operation of one of the two elements of the emitter due to persistent unbalanced operation which was found to contribute to eventual failure by thermal runaway.

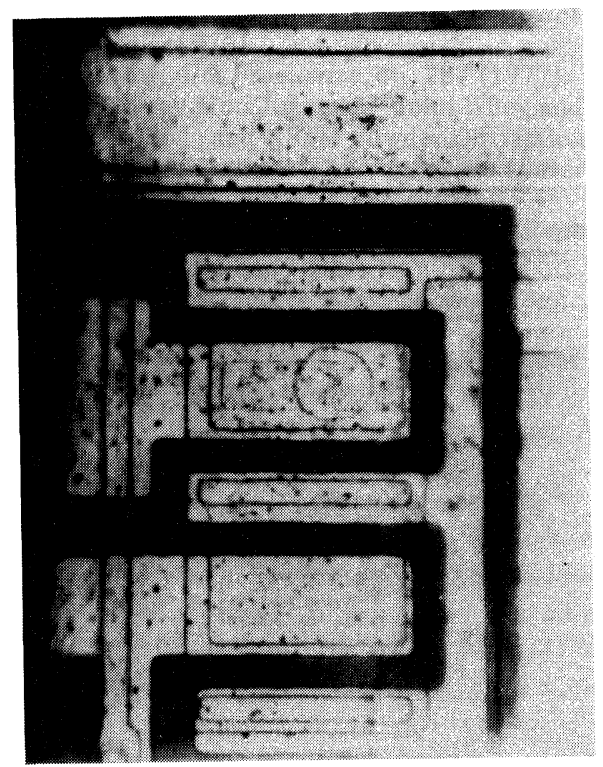

FIGURE 8 Photomicrograph of relay-driver transistor. 


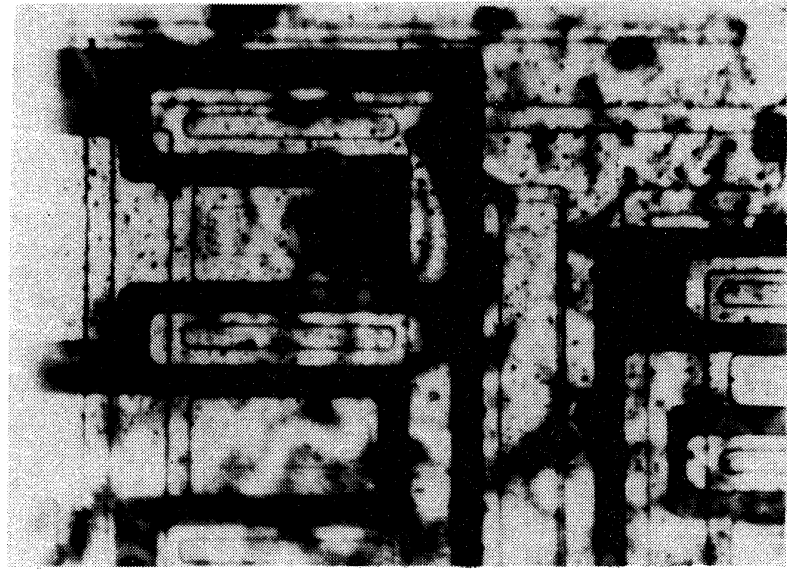

FIGURE 9 Hotter operation of one element of the emitter.

\subsection{Thick-Film Resistors}

In support of current reliability studies, recent attention has been devoted mainly to thick-film resistors whose large planar areas are ideally suited to examination by the technique. The examples presented here are for resistors in arrays in various dual-in-line packages, which were used as test vehicles in the studies. In order to relate thermal ageing during stress tests to the temperatures generated within the resistors, it was necessary to refer to a common parameter such as thermal resistance $\left(R_{\theta}\right)$ - which is defined as the temperature rise per unit power dissipated. Because the measurements showed that the temperature distributions were distinctly non-uniform, and ageing is fastest in the hottest parts, it was decided that the

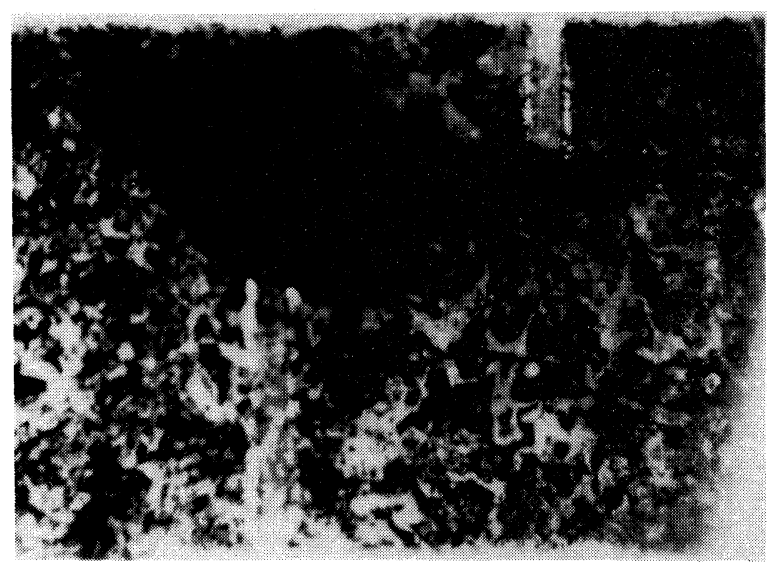

FIGURE 10 Hot spots on thick-film resistors.

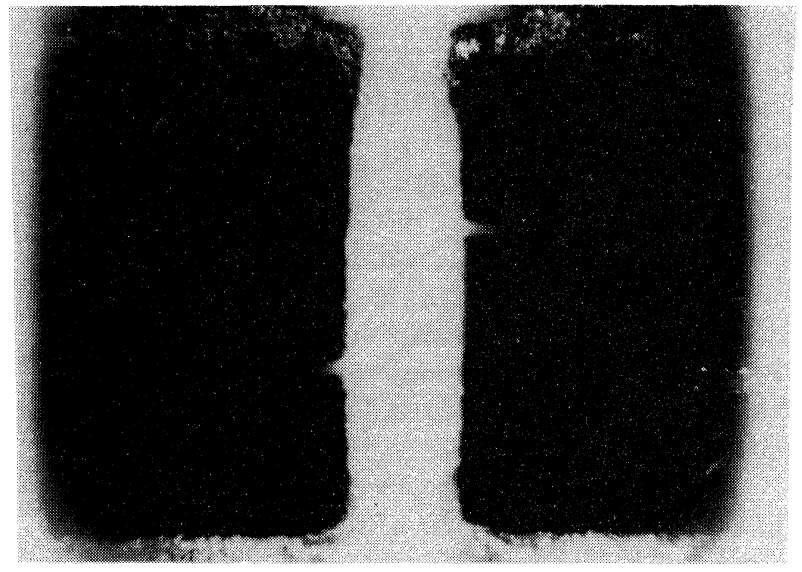

FIGURE 11 Hot spots on thick-film resistor arrays.

thermal resistances should be calculated for the hottest spots on the resistors.

Single resistors gave the most straightforward results because the hot spots occurred at the known sites of maximum dissipation. Illustrated in Figure 10 is a typical observation, that hot spots always originate at the ends of laser "plunge" cuts - the hotter spots occurring at the longer cuts. The values of hot spot thermal resistance for resistors of similar geometry were found to be confined to a small range regardless of their electrical resistivity. Typical thermal resistances were $100^{\circ} \mathrm{C} / \mathrm{W}$ to $125^{\circ} \mathrm{C} / \mathrm{W}$ for $2 \mathrm{~mm} \times 2.2 \mathrm{~mm}$ resistors and $55^{\circ} \mathrm{C} / \mathrm{W}$ to $65^{\circ} \mathrm{C} / \mathrm{W}$ for $2 \mathrm{~mm} \times 4.5 \mathrm{~mm}$ resistors in the range $50 \mathrm{ohm}$ to $50 \mathrm{kohm}$. Generally, resistors located in the middle of substrates had the lower thermal resistances because of the substantial heat sinks surrounding them; but significant variations of $R_{\theta}$ also arose because of differences in the extents of laser cuts in nominally similar resistors.

When power was dissipated in arrays of resistors, the thermal resistances of individual resistors ceased to be meaningful because of the mutual contribution to the generation of heat in the substrate. $R_{\theta}$ then depended on the total power dissipated in the substrate. Hot spots still occurred at the ends of laser cuts in individual resistors (Figure 11), but their location depended on the thickness of the substrate and the configuration of the array. For example, the resistors located in the middle of the substrates were now surrounded by heat sources. Thus, the resistors in which most power was dissipated were not necessarily the hottest. To be consistent with the earlier choice, the thermal resistances were referred once again to the highest temperatures, wherever 


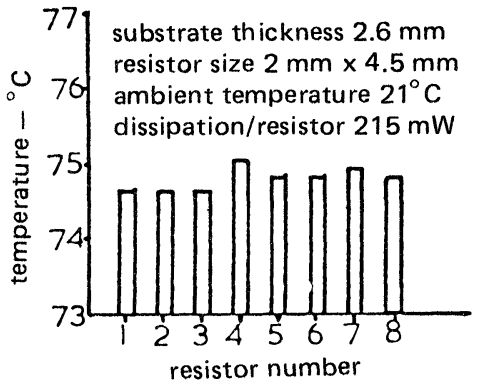

FIGURE 12 Temperature distribution in an array of identical resistors.

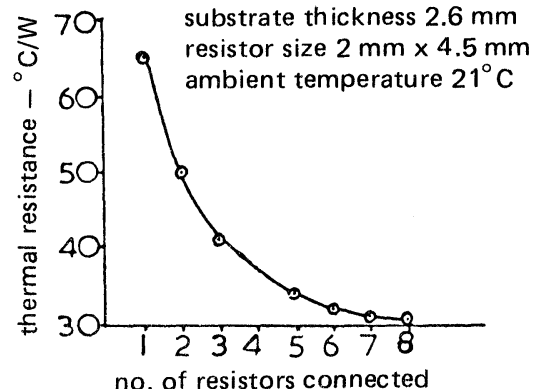

FIGURE 13 Hot spot thermal resistances of resistors in an array.

they occurred in each array. For the simplest case of uniform dissipation in an array of resistors similar in value and geometry, the temperature distributions were found to be uniform, with marginally higher temperatures occurring near the middle of the arrays (Figure 12). Incremental dissipation, successively combining one to eight of the resistors of the array, produced successive decrements in $R_{\theta}$, which tended asymptotically to a constant value (Figure 13) - results that are entirely consistent with the conduction of heat between resistors and the finite boundaries for heat loss from the package. ${ }^{6}$ Even where power was dissipated non-uniformly in an array - likely in applications requiring various resistances in the array - the temperatures were relatively uniform about the centre of the array (Figure 14), endorsing the argument presented earlier of the mutual influence of the dissipations.

The technique has also been used to aid the diagnosis of faults in resistors. Low value resistors, which increased after exposure to voltage surges, were found to have developed hot spots at locations remote from constrictions due to trimming (Figure 15), implying that new constrictions had developed

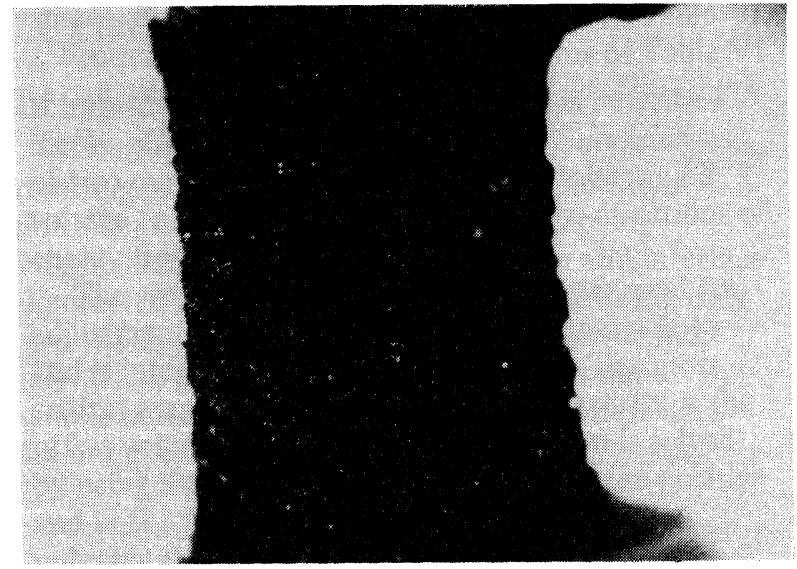

FIGURE 15 Hot spot in damaged resistor.

in the structures. The occurrence of cracks in the resistors, terminating at the location of the hot spots was confirmed by laboriously probing the resistors to produce equipotential contours. The contours in Figure 16 correspond to the resistor in Figure 15 (the crack looks like a laser cut).

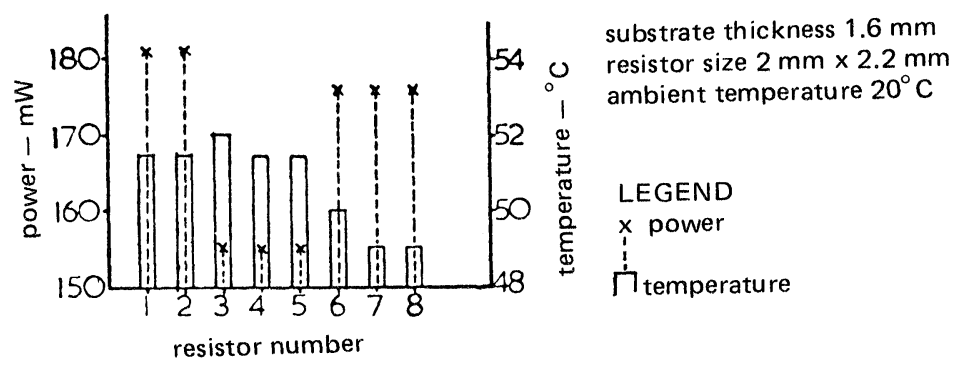

FIGURE 14 Power and temperature distributions in an array of resistors. 


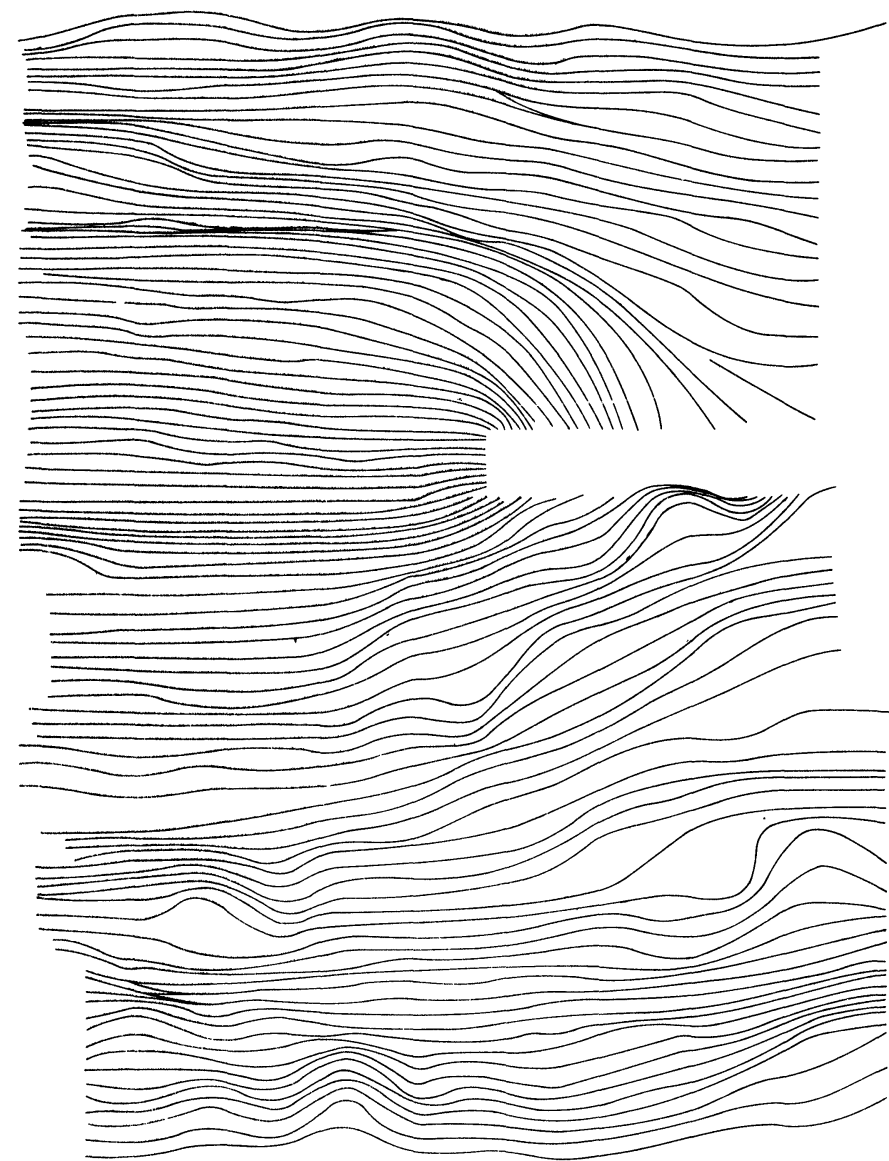

FIGURE 16 Equipotentials in damaged resistor.

\section{DISCUSSION AND CONCLUSIONS}

The foregoing illustrations show that the liquid crystal technique is simple yet provides high spatial resolution, giving it distinct advantages over even the more advanced of the alternative methods of temperature measurement of microcircuits. In principle, the method may be applied to any planar horizontal surface and has a ceiling of about $300^{\circ} \mathrm{C}$. The ready application of the technique to both active and passive components that may be used in hybrid microcircuits, and the obvious benefits of simultaneous visual examination of entire components have been illustrated by the examples presented.

Thick-film resistors have been shown to be eminently suitable components for examination by the technique, which was used in support of reliability evaluation. The observations that were made are consistent with heat conduction between the resistors and substrates. This was particularly notable when significantly non-uniform dissipation within a resistor array still produced a fairly uniform temperature distribution.

It was only in single resistors that local dissipation played a significant part - the variation in thermal resistance due to different extents of trimming giving warning that families of resistors could age at different rates because of trimming cut variations within the design rules of some manufacturers.

The applications of the technique extend beyond the supportive role to reliability evaluation. For instance, it is clear that measurements of thermal resistance and temperature distributions can be used to estimate resistance changes associated with the temperature coefficient of resistance, and in calculating any derating adjustments that are niecessary. As the technique becomes more widely used, still more applications may be discovered. 


\section{ACKNOWLEDGEMENTS}

Acknowledgement is made to the Director of Research of the British Post Office Telecommunications HQ for permission to publish this paper.

\section{REFERENCES}

1. L. Hamiter, "Infrared Techniques for the Reliability Enhancement of Microelectronics," SCP and Solid State Technology, Vol. 10, No. 3, March 1967, pp 41-9.

2. F. N. Sinnadurai, "Mechanisms and Modes of Failure in Silicon Planar Semiconductor Devices", MSc Thesis, University of London, 1968, p. 48.
3. J. L. Fergason, "Liquid Crystals in Nondestructive Testing", Appl. Opt., Vol. 7, No. 9, September 1968 , pp. 1729-37.

4. Francis Nihal Sinnadurai, Christopher Edward Stephens and Alan John Melia, "Temperature Measurement Using Liquid Crystals" British Patent No. 1442802 , Filed 19 Feb. 1973.

5. C. E. Stephens, and F. N. Sinnadurai, "A Surface Temperature Limit Detector Using Nematic Liquid Crystals, With an Application to Microcircuits," J. Sci. Instrum., Vol. 7, 1974, pp. 641-43.

6. K. Board, "Thermal Properties of Annular and Array Geometry Semiconductor Devices on Composite Heat Sinks," Solid-State Electron. Vol. 16, 1973, pp. 1315-20. 

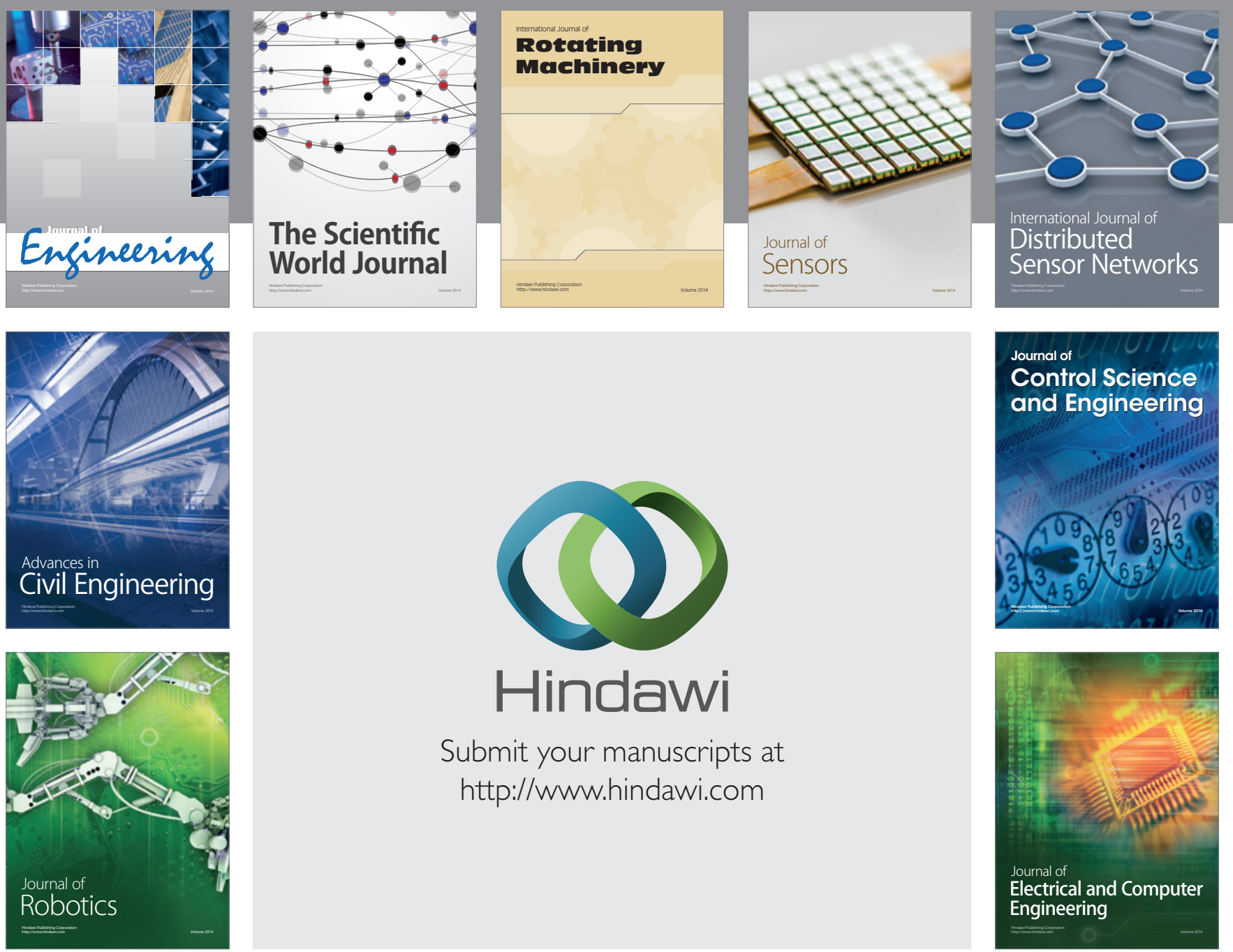

Submit your manuscripts at

http://www.hindawi.com
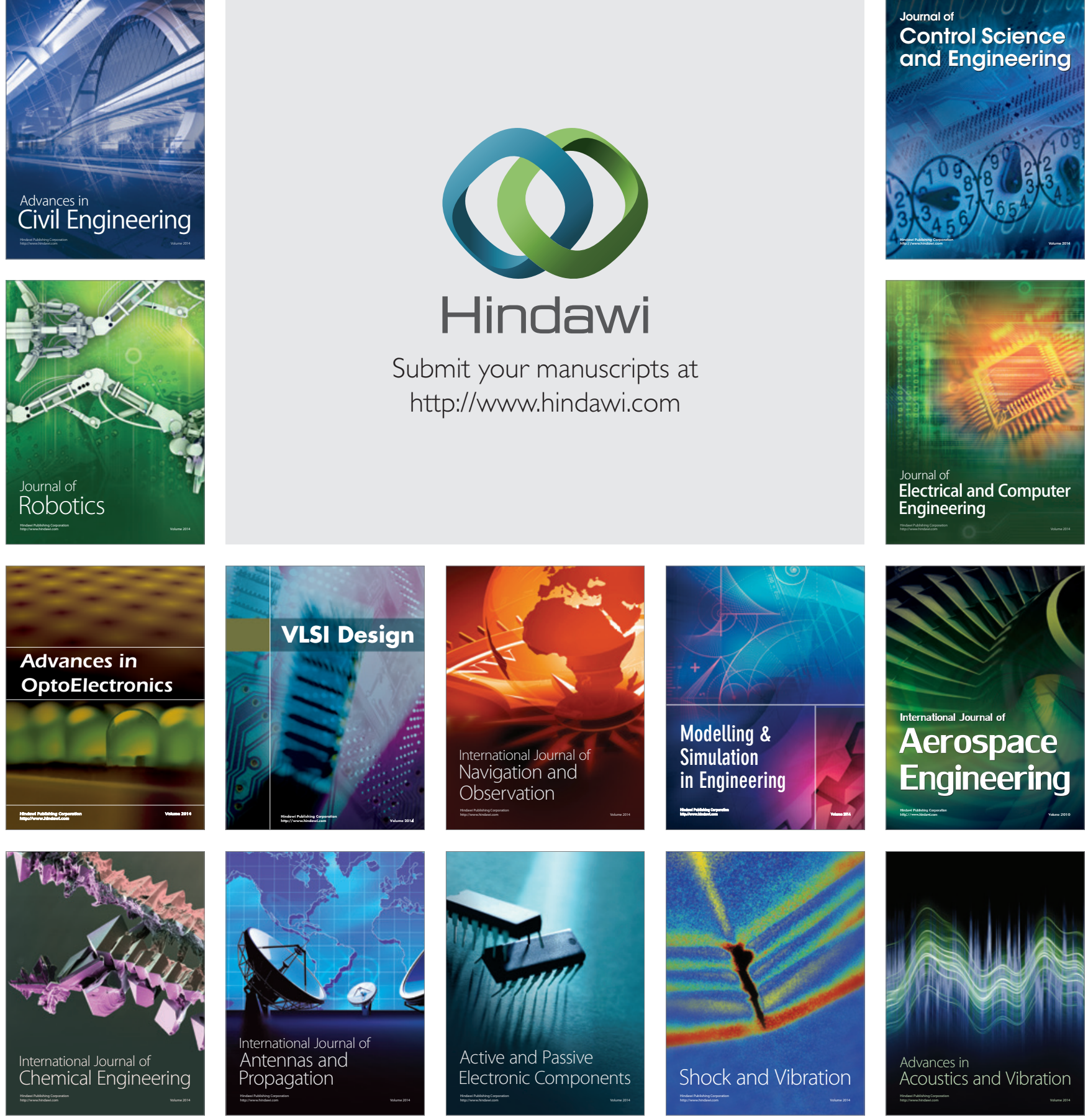